\title{
A case of cryptogenic pseudocirrhosis causing acute liver failure: when clinic and radiology work together
}

\author{
Martina Finocchi, Ombretta Para, Giacomo Zaccagnini, Lorenzo Corbo, Lucia Maddaluni, Margherita Giampieri, \\ Eleonora Blasi, Mattia Ronchetti, Carlo Nozzoli
}

Internal Medicine Department, Careggi University Hospital, Firenze, Italy

\begin{abstract}
It is known that a wild spectrum of hepatic manifestations can be common presentations of metastatic breast cancer. Pseudocirrhosis pattern has been often described as almost always secondary to systemic chemotherapy and it is defined by morphological liver changes that mimic cirrhosis including capsular retraction, nodularity, parenchyma atrophy and caudate lobe, radiologically identifiable. Acute liver injury is an occasional complication in oncologic patients, and it outlines an organ failure when there is evidence of encephalopathy and coagulopathy (international normalized ratio $>1.5$ ) in the absence of pre-existing liver disease, with an illness of $<26$ weeks duration. The two most common etiologies are leukemia/lymphoma followed by breast cancer but also in this case, liver is involved almost always after chemotherapy, hormonotherapy or radiotherapy. Here we present a case of rapid evolving acute liver failure presented as cryptogenic pseudocirrhosis without any evidence of primitive breast cancer but an incidental demonstration.
\end{abstract}

\section{Introduction}

It is known that a wild spectrum of hepatic manifestations can be common presentations of metastatic breast cancer. ${ }^{1}$ Pseudocirrhosis pattern has been often described as almost always secondary to systemic chemotherapy and it is defined by morphological liver changes that mimic cirrhosis including capsular retraction, nodularity, parenchyma atrophy and caudate lobe, radiologically identifiable., ${ }^{2,3}$

Acute liver injury is an occasional complication in oncologic patients, and it outlines an organ failure when there is evidence of encephalopathy and coagulopathy

Correspondence: Ombretta Para, Internal Medicine Department, Careggi University Hospital, Largo Brambilla 3, Pad. 12 sez. G, Firenze, Italy.

Tel.: +39.0557946411. E-mail: ombretta.para@gmail.com

Key words: Acute liver failure; breast cancer; pseudocirrhosis.

Contributions: the authors contributed equally.

Conflict of interests: the authors declare no conflict of interests.

Received for publication: 30 December 2018.

Accepted for publication: 8 February 2019.

This work is licensed under a Creative Commons Attribution NonCommercial 4.0 License (CC BY-NC 4.0).

CCopyright M. Finocchi et al., 2019

Licensee PAGEPress, Italy

Italian Journal of Medicine 2019; 13:121-123

doi:10.4081/itjm.2019.1129 [international normalized ratio (INR) $>1.5$ ] in the absence of pre-existing liver disease, with an illness of $<26$ weeks duration. The two most common etiologies are leukemia/lymphoma followed by breast cancer ${ }^{4}$ but also in this case, liver is involved almost always after chemotherapy, hormonotherapy or radiotherapy.

Here we present a case of rapid evolving acute liver failure presented as a cryptogenic pseudocirrhosis without any evidence of primitive breast cancer but an incidental demonstration.

\section{Case Report}

A 46-year-old otherwise healthy woman came to our emergency department for one-month lasting worsening abdominal pain. At clinical examination of the abdomen, we suspected the presence of ascites and the patient referred hyperchromic urine, normocholic stool and itch. She reported taking contraceptive pill; she denied alcohol consumption and previous blood test showed normal lipidemic profile. Before the admission to our department, chest X-ray (resulted normal) and abdominal ultrasound were performed and ascites was confirmed. The liver structure was suggestive for cirrhosis like subversion of the parenchyma with pseudonodular areas without intra- or extra-hepatic bile ducts dilatation (Figure 1). An hypoechogenic area was found in the uterus, but it was ascribable to a recent myomectomy, performed few months before. Blood tests showed sign of cholestasis (total bilirubin $3.3 \mathrm{mg} / \mathrm{dL}$, direct bilirubin 1.10 , gamma-glutamyltransferase $192 \mathrm{U} / \mathrm{L}$, alkaline phosphatase 271), alanine transaminase $68 \mathrm{U} / \mathrm{L}$, aspartate 
transaminase $122 \mathrm{U} / \mathrm{L}$ and signs of liver failure (INR 1.5 , albumin $26.1 \mathrm{~g} / \mathrm{L}$, cholinesterase $4.414 \mathrm{U} / \mathrm{L}$, ammonium $64 \mathrm{mcmol} / \mathrm{L}$ ). Inflammatory markers were increased (erythrocyte sedimentation rate $66 \mathrm{~mm} / \mathrm{h}$, C-reactive protein $62 \mathrm{mg} / \mathrm{L}$, procalcitonin 0.86 $\mathrm{ng} / \mathrm{mL}$ ). In order to rule out infective causes we searched for major and minor hepatitis virus (hepatitis $\mathrm{A}, \mathrm{B}, \mathrm{C}$ and $\mathrm{E}$, cytomegalovirus and Epstein-Barr virus), HIV, Echinococcus and Entamoeba but serologies were negatives. A neoplastic etiology strengthened, so we asked for a total body contrast computed-tomography (CT) that did not show any thoracic or mediastinal lesion, including normal nodes profile; abundant ascites was confirmed and it was highlighted peritoneal diffusion throw thoracic diaphragm. Liver appeared subverted like in cirrhosis, with several focal points suspected for neoplasm. Intra and extra hepatic bile ducts were regular and not dilated and there was no bone metastasis. We still suspected the uterus to be involved because of the presence of multiple focal lesions, however transvaginal ultrasound confirmed just a fibromyomatosis. An esophagogastroduodenoscopy was performed and it was normal. At this point, the main suspicion was a bile duct neoplasm or a primitive liver cancer so we performed a magnetic resonance cholangiopancreatography but cholangiography sequences were not diagnostic due to the patient's noncompliance and the consistent ascites effusion. Anyway, a desmoplastic reaction in the liver parenchyma was better documented, once again in the absence of bile duct dilatations, no pancreas, kidney or nodal involvement. Surprisingly in the thoracic scans, several vertebral lesions were showed together with two spiculated left breast lesions $(6$ and $10 \mathrm{~mm})$ with contrastographic enhancement. Considering the complexity of the case, we dosed neoplastic markers, confirming the possibility of a primary breast cancer (Ca 15-3: 9407, Ca 125: 1.267, CEA: 11, Ca 19-9: 4). Meantime we performed a liver biopsy, which gave us the chance to make diagnosis of glandular neoplasm with sclerosis (Figures 2 and 3) (CK7 positive, HSA and CD10 negative). Unfortunately, the patient died the day before breast biopsy for the complication of an elapsing sepsis.

\section{Discussion}

Pseudocirrhosis is a radiological diagnosis describing diffuse hepatic nodularity in the absence of a primary reasonable etiology and without histopathological confirmation of cirrhosis.

Breast cancer with liver metastasis treated with chemotherapy, hormonotherapy or radiotherapy is the most commonly reported cause of pseudocirrhosis ${ }^{3}$ but this has also been reported in association with other metastatic diseases, including pancreatic cancer, colon cancer, medullary thyroid cancer, and esophageal cancer..$^{5-8}$

Neoplastic pseudocirrhosis developed without any kind of specific therapy, has been rarely reported as a complication during breast disease progression; 5,9 searching on Medline, there is just another reported case of cirrhosis-like first presentation and postmortem primitive cancer diagnosis. ${ }^{10}$

In both cases a desmoplastic reaction surrounding infiltrating hepatic metastatic masses leads to complication from portal hypertension, that is the most

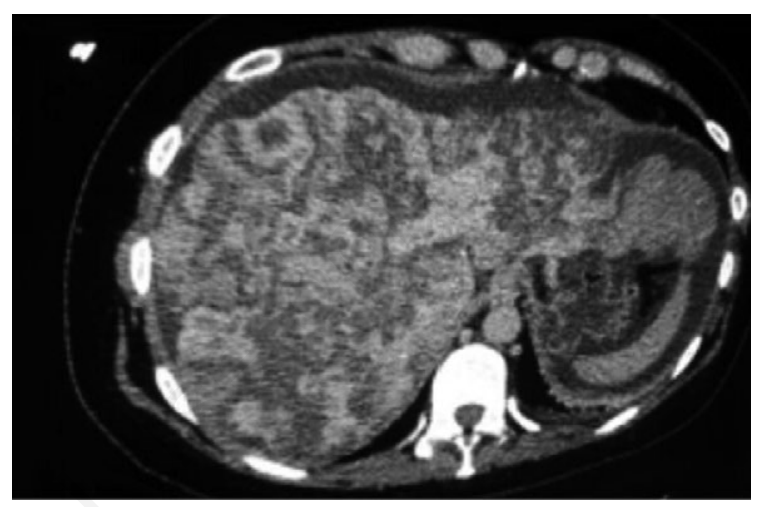

Figure 1. Total subversion of liver parenchyma.

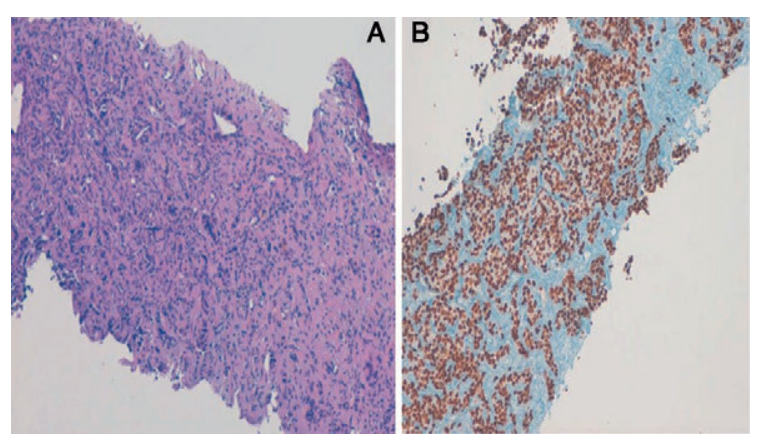

Figure 2. A and B) GATA 3 positivity in liver biopsy.



Figure 3. A) Er+; B) Progesterone+. 
common presentation also in after-therapy hepatic involvement. $^{3}$

Pathophysiology is still unclear: cancer stem cells can acquire metastatic ability and by recruiting vascular endothelial growth factor receptors-positive hemopoietic progenitor cells, they could create premetastatic niches in target organs, rich in fibronectin. ${ }^{11}$ In those cases, presented after chemotherapy, it is assumed that the hepatotoxic effect of systemic chemotherapy or a response to chemotherapy by tumor tissues lead to nodular regenerative hyperplasia, compression, atrophy through tumor shrinkage, and finally fibrosis. ${ }^{5}$ It is important to highlight that nowadays there is no correlation between a specific drug class and the chance of developing pseudocirrhosis. ${ }^{3}$ More studies are needed to clarify the underlying mechanisms.

In our patient the most important clinical feature was a rapidly evolving liver failure, which required a rapid diagnosis. Most reported cases of acute liver failure from metastatic breast cancer are reported in patients with history of known and treated breast cancer. ${ }^{12}$ Moreover our challenge was made more difficult since our patient had no nodes involvement, and this strengthened the suspicion of a ductal or hepatic primitive disease. The radiological evidence of peritoneal diffusion through thoracic diaphragm, could suggest a specific metastatic pattern, ${ }^{13}$ which is not described in other comparable reported cases. In patients without any prior history of cancer, acute liver failure of indeterminate etiology can dangerously delay diagnosis, often ending with patient's death. In this setting histopathological examination and positron-emission tomography-CT may be helpful if driven by clinical suspicion.

\section{Conclusions}

This case wants to focus clinicians' attention on cryptogenic cirrhosis and acute liver failure of unknown etiology, pointing out the importance of an interdisciplinary management of complex cases, with special regard to radiologist and pathologist. More studies are needed to clarify the mechanism of this particular setting of metastasis in the history of primitive breast cancer.

\section{References}

1. Diamond JR, Finlayson AR, Borges VF. Hepatic complications of breast cancer. Lancet Oncol 2009;10:615-21.

2. Jüngst C, Krämer J, Schneider G, et al. Subacute liver failure by pseudocirrhotic metastatic breast cancer infiltration. Ann Hepatol 2013;12:834-6.

3. Adike A, Karlin N, Carey EJ. Pseudocirrhosis: a case series and literature review. Case Rep Gastroenterol 2016;10:381-91.

4. Rich NE. Malignant infiltration of the liver presenting as acute liver failure. Clin Gastroenterol Hepatol 2015;13:1025-8.

5. Lee SL, Chang ED, Na SJ, et al. Pseudocirrhosis of breast cancer metastases to the liver treated by chemotherapy. Cancer Res Treat 2014;46:98-103.

6. Kang SP, Taddei T, McLennan B, Lacy J. Pseudocirrhosis in a pancreatic cancer patient with liver metastases: a case report of complete resolution of pseudocirrhosis with an early recognition and management. World J Gastroenterol 2008;14:1622-4.

7. Kobashigawa C, Nakamoto M, Hokama A, et al. Pseudocirrhosis in metastatic esophageal cancer. South Med J 2010;103:488-9.

8. Battisti S, Guida FM, Pagliara E, et al. Pseudocirrhosis after anti-EGFR-based neoadjuvant therapy for hepatic metastasis from colon cancer: adifferent point of view. Clin Colorectal Cancer 2014;13:e13-5.

9. Jüngst C, Krämer J, Schneider G, et al. Subacute liver failure by pseudocirrhotic metastatic breast cancer infiltration. Ann Hepatol 2013;12:834-6.

10. Sass DA, Clark K, Grzybicki D, et al. Diffuse desmoplastic metastatic breast cancer simulating cirrhosis with severe portal hypertension: a case of "pseudocirrhosis". Dig Dis Sci 2007;52:749-52.

11. Lu X, Kang Y. Organotropism of breast cancer metastasis. J Mammary Gland Biol Neoplasia 2007;12:153-62.

12. Nazario HE, Lepe R. Metastatic breast cancer presenting as acute liver failure. Gastroenterol Hepatol 2011;7:1.

13. Davidson B. Biological characteristics of cancers involving the serosal cavities. Crit Rev Oncog 2007;13: 189-227. 